
\section{$\zeta_{\text {six }}$}

'Division of Thoracic Surgery, Department of Surgery, Taipei Veterans General Hospital, Taipei, Taiwan

${ }^{2}$ Department of Surgery, National Yang-Ming University Hospital, Yilan, Taiwan ${ }^{3}$ Department of Radiology, Taipei Veterans General Hospital, Taipei, Taiwan

${ }^{4}$ AIDS Prevention and Research Center, National Yang-Ming University, Taipei, Taiwan Diseases, Department of Internal Medicine, Taipei Veterans General Hospital, Taipei, Taiwan

${ }^{6}$ National Yang-Ming University School of Medicine, Taipei, Taiwan

\section{Correspondence to}

Dr Chien-Sheng Huang, Division of Thoracic Surgery, Department of Surgery, Taipei Veterans General Hospital, No 201 Section 2 Shih-Pai Road, Taipei 112, Taiwan; huangcs@ vghtpe.gov.tw

Received 3 April 2012

Accepted 11 June 2012 Published Online First 26 June 2012

To cite: Hsieh M-S, Chen C-K, Wong W-W, et al. Thorax 2013;68:307308. ${ }^{5}$ Division of Infectious

\section{Rapid-growth pneumatocele mimics massive pneumothorax in a HIV-positive patient}

\author{
Min-Shiau Hsieh, ${ }^{1,2}$ Chun-Ku Chen, ${ }^{3}$ Wing-Wai Wong, ${ }^{4,5}$ Chien-Sheng Huang ${ }^{1,6}$
}

We present a rare fast-growing giant pneumatocele in a patient presenting with suspected pneumocystis pneumonia (PCP) infection and bilateral pneumothoraces as a primary manifestation of AIDS (HIV viral loading test: 628000 copies $/ \mathrm{ml}$ ). Tube thoracostomies were performed and complicated with enduring air leakage and subcutaneous emphysema. Follow-up chest $\mathrm{X}$-rays showed an enlarging radiolucency over the left upper lung field that was interpreted as massive pneumothorax with passive lung atelectasis. Positive ventilation was also applied due to disease progression (The $\mathrm{CD}^{+}$T-lymphocyte count was $18 / \mathrm{cu} \mathrm{mm}$ ). Repeated chest CT scans disclosed a newly formed giant bullous-liked lesion in the left upper lung field (figure 1). Video-assisted thoracoscopic surgery for unroofing the cystic lesion (pneumatocele) and pleurodesis successfully allowed the patient to wean from the ventilator and be discharged uneventfully (figure 2).

\section{DISCUSSION}

HIV with PCP infection complicated with necrotising alveolitis in the subpleural pulmonary

\section{Learning points}

A fast-growing giant pneumatocele can develop in the HIV-positive patient with suspected PCP infection complicated with pneumothorax and compromised pulmonary reserve. CT scans might be helpful for differential diagnosis.

- Surgical intervention by video-assisted thoracoscopic surgery for unroofing the pneumatocele and pleurodesis might be an effective treatment to resolve the respiratory comprise and pneumothorax.

parenchyma that resulted in pneumothorax and pneumatocele have been well reported. ${ }^{1}{ }^{2}$ Nonetheless, a rapid-growth giant pneumatocele could be misinterpreted as massive pneumothorax

Contributors M-SH was involved in collecting clinical information and drafting the manuscript. C-CK analysed the radiological studies. without expectation.

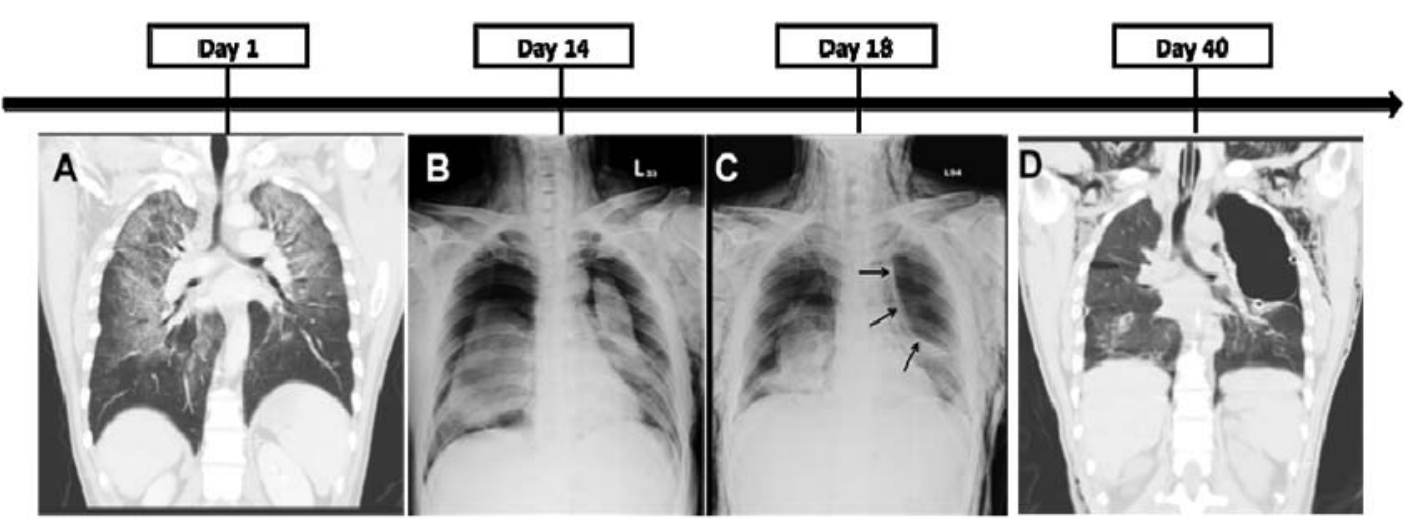

Figure 1 (A) Chest CT scan on Day 1: showed symmetric bilateral perihilar ground-glass opacity with predominant upper and middle lung zone involvement; (B) chest radiograph on Day 14 showed spontaneous bilateral pneumothoraces; (C) chest radiograph on Day 18 depicted enlarging radiolucent area in bilateral lung fields with further collapse of both lungs and extension of subcutaneous emphysema, progressive pneumothorax with passive lung atelectasis; arrows indicated the new giant pneumatocele already formed; (D) repeated chest CT disclosed a giant cystic lesion in the left lung. This figure is only reproduced in colour in the online version. 

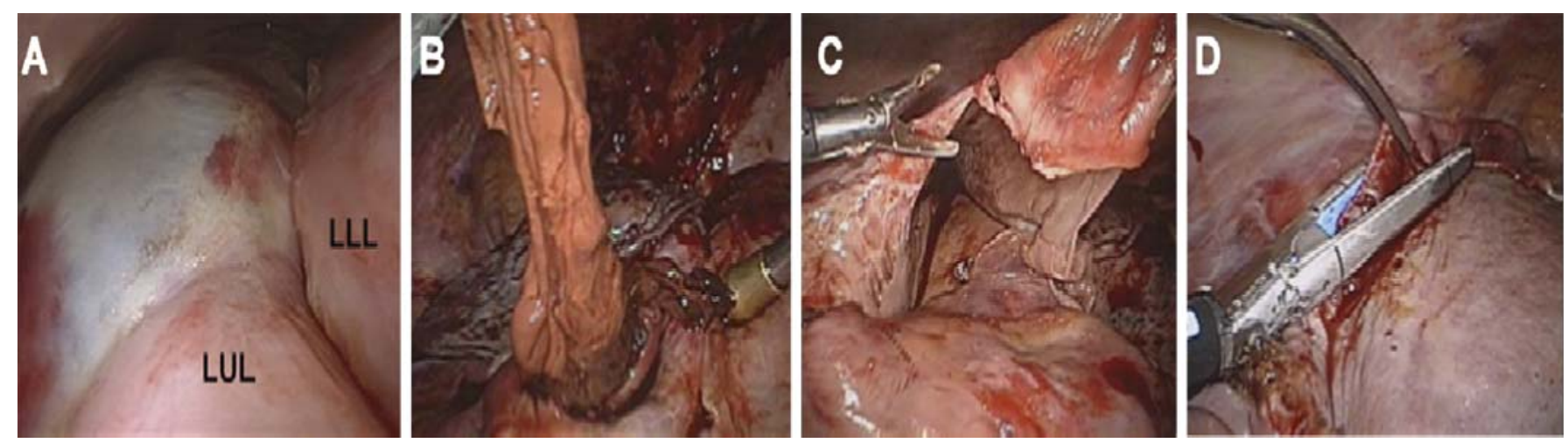

Figure 2 Intraoperative findings: (A) a giant bullous lesion (pneumatocele) compromised the residual pulmonary parenchyma; (B) necrotic debris inside the pneumatocele which revealed only coagulase negative staphylococcus species infection; $(C)$ unroofing the pneumatocele by video-assisted thoracoscopic surgery; (D) trimming of the margin of pneumatocele with healthy parenchyma to minimise the air leakage. This figure is only reproduced in colour in the online version.

WW-W carried out the patient care clinically and helped to draft the manuscript. C-SH provided the operative images and wrote the manuscript.

\section{Competing interests None.}

Patient consent Obtained.

Provenance and peer review Not commissioned; externally peer reviewed.

\section{REFERENCES}

1 MacDuff A, Arnold A, Harvey J. Management of spontaneous pneumothorax: British Thoracic Society Pleural Disease Guideline 2010. Thorax 2010;65(Suppl 2):ii18-31.

2 Huang L, Cattamanchi A, Davis JL, et al. HIV-associated Pneumocystis pneumonia. Proc Am Thorac Soc 2011;8:294-300. 